Vol 12, Issue 1, 2019

\title{
PHARMACOLOGICAL TREATMENT OF OSTEOPOROSIS IN PATIENTS WITH CORONARY HEART DISEASE COMPLICATED BY CHRONIC HEART FAILURE
}

\author{
MARIYA MARUSHCHAK*, INNA KRYNYTSKA \\ Department of Functional and Laboratory Diagnostics, I. Horbachevsky Ternopil State Medical University, Ukraine. \\ Email: marushchak@tdmu.edu.ua
}

Received: 23 August 2018, Revised and Accepted: 21 November 2018

\begin{abstract}
Objective: Chronic heart failure (CHF) is a chronic multisystem disorder associated with a myriad of metabolic disturbances. Moreover, CHF may adversely affect bone metabolism and induce a severe bone loss, increasing susceptibility to fractures and osteoporosis. This study investigates the effect of combined calcium with Vitamin D3 supplement called "calcemin advance" and calcitonin in the prophylaxis and treatment of osteopenia and osteoporosis in patients with coronary heart disease (CHD) complicated by CHF with the establishment of their influence on the bone mineral density (BMD).
\end{abstract}

Methods: A total of 59 patients with CHD complicated by CHF. Interventions: The physical examination findings, dual-energy X-ray absorptiometry findings, and treatment results were assessed.

Results: The results of the calcemin advance usage in patients with osteopenic changes showed a positive dynamics of the studied parameters, both in the lumbar spine and in the femoral bone. In patients with osteoporosis, the usage of osteoprotective therapy (calcemin advance+miacalcic) contributed to the increasing of BMD in the lumbar spine and in the femoral bone versus patients who received only combined calcium with Vitamin D3 supplement.

Conclusions: Our results suggest that bone density screening could be recommended in patients with prevalent CHF. Moreover, the results of our investigation substantiate the necessity and effectiveness of osteoprotective therapy in patients with CHF with osteoporosis by calcitonin (Miacalcic), in combination with combined calcium and Vitamin D3 supplement (calcemin advance), and by only calcemin advance - in patients with CHF and osteopenia.

Keywords: Chronic heart failure, Osteopenia, Osteoporosis, Therapy.

(C) 2019 The Authors. Published by Innovare Academic Sciences Pvt Ltd. This is an open access article under the CC BY license (http://creativecommons. org/licenses/by/4. 0/) DOI: http://dx.doi.org/10.22159/ajpcr.2019.v12i1.29293

\section{INTRODUCTION}

Cardiovascular diseases (CVDs) remain the biggest cause of deaths worldwide [1-3]. It accounts for $45.1 \%$ of mortality, the majority of this in the form of coronary heart disease (CHD) [4]. In 2015, 19.9 million CVDs deaths occurred (one-third of all global deaths), and 423 million people had prevalent CVDs ( $\sim 1$ in 17 of the global population) [5,6]. By 2020, 7 of 10 deaths in developing countries are predicted to be due to noncommunicable diseases - the majority of which would be due to CVDs [7]

Chronic heart failure (CHF) is a complex clinical syndrome in which abnormal heart function results in, or increases the subsequent risk of, clinical symptoms and signs of reduced cardiac output, and/or pulmonary or systemic congestion at rest or with stress [8]. CHF is, nowadays, an important health problem. Not only is CHF associated with high use of resources and health-care cost but also the prevalence of heart failure is increasing due to better care and treatment and to the aging of the population [9]. The prevalence of CHF is $\sim 2 \%$ of the general population in the developed countries, increasing to $\sim 10 \%$ among people $>70$ years of age; moreover, the prevalence increased with age in both men and women. Worldwide, the number of patients with heart failure has reached 22.5 million [10].

CHF is a chronic multisystem disorder associated with a myriad of metabolic disturbances. Moreover, CHF may adversely affect bone metabolism and induce a severe bone loss, increasing susceptibility to fractures and osteoporosis [11]. It should be noted that CHF and osteoporosis are often diagnosed in the same patient [12].
Therefore, both CHF and osteoporosis are common causes of loss of function and independence and have become a heavy burden on the health-care system. Our previous studies showed that structural and functional changes in the bone tissue of the lumbar spine were found in $75.9 \%$ of patients with Stage 1 of CHF caused by ischemic heart disease: $64.4 \%$ of patients had osteopenic syndrome and $11.5 \%$ - osteosclerotic changes. In $75.8 \%$ of CHF patients, we have found structural and functional changes in the bone tissue of proximal right femur [13]. Structural and functional changes of bone tissue of the lumbar spine have been found in $49.2 \%$ of patients with CHD complicated by Stage II-A CHF, in particular, I stage of osteopenia - in $44.6 \%$, II stage of osteopenia - in $27.7 \%$, III stage of osteopenia - in 10.8\%, and osteoporosis - in 16.9\% [14].

\section{Objectives}

This study aims to investigate the effect of combined calcium with Vitamin D3 supplement called "calcemin advance" and calcitonin in the prophylaxis and treatment of osteopenia and osteoporosis in patients with CHD complicated by CHF with the establishment of their influence on the bone mineral density (BMD).

\section{METHODS}

Subject selection

The study involved 59 patients with CHD complicated by CHF according to the classification by N.D. Strazhesko, V.H. Vasilenko and G.F. Lung (1935) at Ternopil University Hospital.

The average age of patients was $57.91 \pm 9.30$ years. Their body mass index exceeded normal range (set at $<25 \mathrm{~kg} / \mathrm{m}^{2}$ ) and was in the range of subcompensated obesity $-28.04 \pm 2.12 \mathrm{~kg} / \mathrm{m}^{2}$. Clinical characteristics of the 
patients were as follows: The disease duration ranged from 2 to 20 years, the underlying disorder mainly was cardiosclerosis. In patients' history was found bad habits: 16 - smoking, 4 - alcohol abuse, and 12 - caffeine abuse (4-5 cups of coffee per day). A history of fractures was found in 9 persons.

The patients did not have other severe comorbidities that could have caused changes in bone tissue. A total of 59 participants were used for this research work. Informed written consent was provided by all participants. Ethical approval was obtained from I. Horbachevsky Ternopil State Medical University Ethics Committee.

Diagnosis of CHD was confirmed using a set of characteristic anamnestic, clinical (typical angina attacks with physical and psychoemotional stress), biochemical (increased total cholesterol and total lipids levels), and electrocardiography (ST-segment depression below the baseline, T-wave inversion) data. To confirm the diagnosis, we also used data obtained during a physical examination, such as enlarged to the left heart boundaries, weakened sound of the top tone, stress of the second tone above the aorta, changes in blood pressure and heart rate.

Toverify the diagnosis of heart failure we have used the main limiting factors of physical performance and clinical symptoms: Dyspnea, tachycardia, and fatigue after exertion. The final diagnosis of $\mathrm{CHF}$ with systolic dysfunction was given based on the results of echocardiography test.

\section{Determination of BMD}

Dual-energy X-ray absorptiometry is widely used for measuring BMD due to its recognized precision. The method utilizes two measurements, T-score and Z-score. The first one is calculated when the patient's BMD is subtracted from mean BMD of a population of healthy young adults, matched. The T-score is calculated as a number of standard deviations (SDs); the patient's measured BMD is above or below the mean for population of healthy 30-year adults, matched for sex and ethnicity. The Z-score is expressed in units of the population SD, but instead of comparing the patient's BMD to the mean of the young adult population, it is compared with the mean BMD of a healthy population matched for age, sex, and ethnicity. In this study, we evaluated the scores according to the WHO guidelines (WHO, Geneva, 1994): BMD $>1.2 \mathrm{~g} / \mathrm{sm}^{2}$ is classified as osteosclerosis; a T-score $\geq-1$ is regarded as normal, and $\mathrm{T}$-score between -2.5 and -1 is classified as osteopenia [15].

Standard therapy for patients with CHF included the use of an angiotensin-converting enzyme inhibitor, $\beta$-adrenoblocker, diuretic, and cardiac glycoside according to the recommendations of the Ukrainian Association of Cardiologists for the diagnosis, treatment, and prevention of CHF. Patients' therapy was as standardized as possible. Before the survey, patients did not receive antiresorptive drugs for the prophylaxis and treatment of osteoporosis.

To study the effects of the combined calcium with Vitamin D3 supplement (calcemin advance) and calcitonin (Miacalcic) on bone tissue, the patients were distributed as follows. We have used Calcemin Advance (Sagmel, Inc. USA), which consists of calcium carbonate $1312 \mathrm{mg}$, Vitamin D3 $200 \mathrm{IU}$, magnesium oxide $40 \mathrm{mg}$, zinc oxide $7.5 \mathrm{mg}$, copper $1.0 \mathrm{mg}$, and sodium borate $2.45 \mathrm{mg}$

The $1^{\text {st }}$ Group (12 persons) consisted of patients with normal BMD, which was carried out only standardized therapy.

The $2^{\text {nd }}$ Group of patients (13 persons) received the same standardized therapy as the $1^{\text {st }}$ Group, but there BMD was characterized by osteopenic changes.

The $3^{\text {rd }}$ Group of patients (15 persons) with osteopenic changes, in addition to standard therapy, was prescribed combined calcium with Vitamin D3 supplement - calcemin advance. Calcemin advance therapy was performed for 3 months ( 1 tablet 2 times per day).

The $4^{\text {th }}$ Group of patients (10 persons) received the same therapy as the third one, but changes in bone tissue were consistent with osteoporosis.
The $5^{\text {th }}$ Group of patients ( 9 persons) with diagnosed osteoporosis received the same therapy as the $4^{\text {th }}$ Group, and calcitonin (Miacalcic). Miacalcic (salmon calcitonin, Switzerland) was administrated according to the scheme, depending on the degree of osteogenic changes (100 IU per day intramuscularly every other day during the $1^{\text {st }}$ month, $200 \mathrm{IU}$ per day every day intranasal $-2^{\text {nd }}$ and $3^{\text {rd }}$ month). In parallel, patients received calcemin advance for 1 tablet 2 times per day for 3 months. The effectiveness of therapy with was evaluated after 3 months.

\section{Data analysis}

The results were analyzed using Statistica 7.0 software and presented as mean with SDs. To evaluate the distribution of the character together by sampling data, we have used Lilliefors and Kolmogorov-Smirnov tests. The differences between all groups were determined using one-way ANOVA, followed by post hoc least significant difference test. $\mathrm{p}<0.05$ was considered statistically significant.

\section{RESULTS}

The analysis of the therapy effectiveness showed that standard therapy does not decrease bone resorption processes, and leads to loss of $1.6 \%$ of bone mass in patients with normal BMD and 3.8\% in patients with osteopenic changes. The results of the calcemin advance usage in patients with osteopenic changes showed a positive dynamics of the studied parameters, both in the lumbar spine and in the femoral bone. Thus, an additional therapy with calcemin advance in patients with CHF and osteopenia of different stage caused a statistically significant increase in BMD in the first two lumbar vertebrae with a simultaneous decrease in the manifestations of the toxic component and pain

Table 1: BMD of lumbar vertebrae in patients with CHF and osteopenia, before and after therapy

\begin{tabular}{lllll}
\hline Status & $\mathbf{L}_{1}$ & $\mathbf{L}_{2}$ & $\mathbf{L}_{3}$ & $\mathbf{L}_{4}$ \\
\hline $2^{\text {nd }}$ Group & & & & \\
$\quad$ Before therapy & $1.02 \pm 0.02$ & $1.10 \pm 0.03$ & $1.12 \pm 0.02$ & $0.98 \pm 0.03$ \\
$\quad$ After therapy & $0.96 \pm 0.02^{*}$ & $1.06 \pm 0.03$ & $1.05 \pm 0.02^{*}$ & $1.02 \pm 0.03$ \\
$3^{\text {rd }}$ Group & & & & \\
$\quad$ Before therapy & $0.97 \pm 0.02$ & $1.05 \pm 0.02$ & $1.10 \pm 0.02$ & $1.12 \pm 0.02$ \\
$\quad$ After therapy & $1.05 \pm 0.03^{*}$ & $1.12 \pm 0.02^{*}$ & $1.14 \pm 0.02$ & $1.15 \pm 0.02$ \\
\hline
\end{tabular}

Results are expressed as a mean and standard deviation. $\mathrm{P}<0.05$ indicates significant differences. BMD: Bone mineral density, CHF: Chronic heart failure, L: Lumbar vertebrae

Table 2: BMD of femoral bone in patients with CHF and osteopenia, before and after therapy

\begin{tabular}{lllll}
\hline Status & Neck & \multicolumn{1}{c}{ Ward triangle } & Trochanter & Total \\
\hline $2^{\text {nd }}$ Group & & & \\
Before therapy & $0.92 \pm 0.02$ & $0.82 \pm 0.03$ & $0.89 \pm 0.03$ & $1.04 \pm 0.02$ \\
After therapy & $0.94 \pm 0.03$ & $0.74 \pm 0.02^{*}$ & $0.90 \pm 0.03$ & $1.04 \pm 0.03$ \\
$3^{\text {rd }}$ Group & & & \\
Before therapy & $0.91 \pm 0.03$ & $0.74 \pm 0.02$ & $0.81 \pm 0.03$ & $0.99 \pm 0.03$ \\
After therapy & $0.94 \pm 0.03$ & $0.82 \pm 0.03^{*}$ & $0.88 \pm 0.02^{*}$ & $1.00 \pm 0.03$ \\
\hline \multicolumn{2}{l}{$\begin{array}{l}\text { Results are expressed as a mean and standard deviation. P<0.05 indicates } \\
\text { significant differences. BMD: Bone mineral density, CHF: Chronic heart failure }\end{array}$}
\end{tabular}

Table 3: BMD of lumbar vertebrae in patients with CHF and osteoporosis, before and after therapy

\begin{tabular}{lllll}
\hline Status & $\mathbf{L}_{1}$ & $\mathbf{L}_{2}$ & $\mathbf{L}_{3}$ & $\mathbf{L}_{4}$ \\
\hline $4^{\text {th }}$ Group & & & & \\
$\quad$ Before therapy & $0.81 \pm 003$ & $0.85 \pm 0.03$ & $0.92 \pm 0.03$ & $0.94 \pm 0.03$ \\
$\quad$ After therapy & $0.75 \pm 0.02$ & $0.84 \pm 0.03$ & $0.90 \pm 0.03$ & $0.92 \pm 0.04$ \\
$5^{\text {th }}$ Group & & & & \\
$\quad$ Before therapy & $0.78 \pm 0.03$ & $0.87 \pm 0.04$ & $0.92 \pm 0.03$ & $0.87 \pm 0.02$ \\
After therapy & $0.87 \pm 0.02^{*}$ & $0.92 \pm 0.04$ & $0.94 \pm 0.03$ & $0.89 \pm 0.03$ \\
\hline
\end{tabular}

Results are expressed as a mean and standard deviation. $\mathrm{P}<0.05$ indicates significant differences. BMD: Bone mineral density, CHF: Chronic heart failure, L: Lumbar vertebrae 
Table 4: BMD of femoral bone in patients with CHF and osteoporosis, before and after the therapy

\begin{tabular}{|c|c|c|c|c|}
\hline Status & Neck & Ward triangle & Trochanter & Total \\
\hline \multicolumn{5}{|l|}{$4^{\text {th }}$ Group } \\
\hline Before therapy & $0.78 \pm 0.04$ & $0.57 \pm 0.04$ & $0.72 \pm 0.04$ & $0.86 \pm 0.05$ \\
\hline After therapy & $0.81 \pm 0.05$ & $0.63 \pm 0.05$ & $0.71 \pm 0.04$ & $0.86 \pm 0.05$ \\
\hline \multicolumn{5}{|l|}{$5^{\text {th }}$ Group } \\
\hline Before therapy & $0.86 \pm 0.02$ & $0.65 \pm 0.01$ & $0.77 \pm 0.03$ & $0.92 \pm 0.03$ \\
\hline After therapy & $0.87 \pm 0.02$ & $0.70 \pm 0.03$ & $0.78 \pm 0.04$ & $0.93 \pm 0.03$ \\
\hline
\end{tabular}

syndrome in the heart failure clinical manifestations (Table 1). Modified therapy with the inclusion of calcemin advance to standard therapy enhances bone mineralization in patients of $3^{\text {rd }}$ Group in the femoral neck by $3.3 \%$, in Ward's triangle - by $10.8 \%$, in greater trochanter by $8.6 \%$, and in proximal femur - by $1.0 \%$ versus BMD indices in these patients before treatment (Table 2).

In patients with osteoporosis, the usage of osteoprotective therapy (calcemin advance+miacalcic) contributed to the increasing of BMD at $\mathrm{L}_{1}$ region by $16.0 \%(\mathrm{p}<0.01), \mathrm{L}_{2}$ - by $9.5 \%(\mathrm{p}<0.05)$ versus patients who received only combined calcium with Vitamin D3 supplement (Table 3).

Modified therapy with the inclusion of calcemin advance and Miacalcic to standard therapy enhances bone mineralization in patients of $5^{\text {th }}$ Group in the femoral neck by $7.4 \%$, in Ward's triangle - by $11.1 \%$, in greater trochanter - by $9.8 \%$, and in proximal femur - by $8.1 \%$ versus BMD indices in patients of $4^{\text {th }}$ Group who received only combined calcium with Vitamin D3 supplement (Table 4).

Furthermore, it should be noted that the rate of BMD reduction and its restoring with the use of osteoprotective therapy depends on the stage of osteodeficiency. Hence, in the presence of osteoporosis patients, lose more bone mass faster, but bone tissue faster is restored with the application of adequate treatment.

\section{DISCUSSION}

Osteoporosis is a systemic metabolic bone disease, which is characterized by decrease of bone mass, as well as degeneration of bone microstructure and is prone to lead to fracture due to increased bone fragility [16]. In Europe, osteoporosis accounts for more disabilityadjusted life years than many non-communicable diseases including rheumatoid arthritis, Parkinson's disease, breast cancer, and prostate cancer [17]. Although plenty of studies on osteoporosis and CVDs have been carried out, there are still some controversies on the findings [18].

Our results and research made by Lewiecki and Silvermanhave indicated that bone micro-architectural changes associated with bone loss are largely irreversible $[19,20]$. Although treatment can increase BMD and reduce the risk of fracture, it is unlikely to fully restore the quality and strength of bone to normal. BMD in adults is determined by peak bone mass (PBM), which is the maximum bone mass achieved in life, and the subsequent rate of bone loss. The prevention of osteoporosis or low BMD is directed to maximizing PBM and minimizing the rate of bone loss, with the ultimate goal of maintaining bone strength and preventing fractures. Stabilizing BMD or reducing the rate of bone loss is the primary objective in the prevention of osteoporosis [20].

Li et al. suggested that patients with osteoporosis have a higher risk of CHD than those without osteoporosis. Patients who have osteoporosis and have received treatment with bisphosphonates have a significantly lower risk for CHD than those without treatment [21]. In the placebo branch of the MORE study, osteoporosis (T-score <-2.5 at the spine or the femoral neck) was associated with a fivefold higher risk of a cardiovascular event (for example, stroke and myocardial infarction). In a group of 6800 men and women (MONICA and Västerbotten Intervention Programme databases), low hip BMD was associated with a higher risk of myocardial infarction [22].
Potential mechanisms for the link between osteoporosis and CVD remain unknown. One hypothesis puts forth that the coexistence of osteoporosis and CVD is due to their shared etiological factors (such as smoking, physical activity, alcohol intake, menopause, and hypertension), which may simultaneously promote or inhibit atherosclerosis and bone demineralization. However, in many epidemiologic studies, the association between osteoporosis and CVD remained even after the adjustment of some of these risk factors. Second, common pathophysiological mechanisms are implicated in the progression of the two conditions: Inflammatory cytokines, endogenous sex hormones, oxidized lipids, Vitamin K deficiency, and Vitamin D. Third, the coexistence of osteoporosis and CVD may be due to common genetic factors. Genome-wide association studies have identified several genes and single nucleotide polymorphisms associated with BMD, and CVD risk factors or metabolic traits [23].

Calcium supplements marginally reduce the risk of fracture, and observational studies suggest that high calcium intake might protect against vascular disease, and the findings are consistent with those of interventional studies of calcium supplements that show improvement in some vascular risk factors [24]. On the another hand, calcification in the aorta and coronary arteries may increase cardiovascular risk through the activation of the bone morphogenetic protein, alkaline phosphatase, osteopontin, and matrix GLA protein [25].

\section{CONCLUSIONS}

Our results suggest that bone density screening could be recommended in patients with prevalent CHF. Moreover, the results of our investigation substantiate the necessity and effectiveness of osteoprotective therapy in patients with CHF with osteoporosis by calcitonin (Miacalcic), in combination with combined calcium and Vitamin D3 supplement (calcemin advance), and by only calcemin advance - in patients with CHF and osteopenia.

\section{AUTHORS' CONTRIBUTION}

M.M. and K.I. conceived and designed the research study. M.M. performed the experiment and data collection. M.M. carried out data analysis and interpretation with support from K.I. K.I. wrote the manuscript and M.M. revised the manuscript critically. M.M. and K.I. made the final approval of the manuscript to be published.

\section{CONFLICTS OF INTEREST}

The authors declare that there are no conflicts of interest regarding the publication of this paper.

\section{REFERENCES}

1. Benjamin EJ, Virani SS, Callaway CW, Chamberlain AM, Chang AR, Cheng S, et al. Heart disease and stroke statistics-2018 update: A report from the American heart association. Circulation 2018;137:e67-e492.

2. Piepoli MF, Hoes AW, Agewall S, Albus C, Brotons C, Catapano AL, et al. 2016 European guidelines on cardiovascular disease prevention in clinical practice: The sixth joint task force of the European society of cardiology and other societies on cardiovascular disease prevention in clinical practice (constituted by representatives of 10 societies and by invited experts)Developed with the special contribution of the European association for cardiovascular prevention and rehabilitation (EACPR). Eur Heart J 2016;37:2315-81. 
3. Logoyda L. A HPLC-MS/MS method development and validation for the simultaneous determination of nifedipine and enalapril in human plasma. Int J Appl Pharm 2018;10:35-42.

4. Stewart J, Manmathan G, Wilkinson P. Primary prevention of cardiovascular disease: A review of contemporary guidance and literature. JRSM Cardiovasc Dis 2017;6:2048004016687211.

5. Mozaffarian D. Global scourge of cardiovascular disease: Time for health care systems reform and precision population health. J Am Coll Cardiol 2017;70:26-8.

6. Demikhova N, Chernatska O, Mazur T, Bokova S, Rudenko T, Bumeister L, et al. Markers of cardiovascular complications in patients with Type 2 diabetes mellitus and arterial hypertension. Bangladesh $\mathrm{J}$ Med Sci 2018;17:319-22.

7. Som S. Deficiencies in global health training for cardiovascular fellows. J Am Coll Cardiol 2017;69:1748-52.

8. Ezekowitz JA, O’Meara E, McDonald MA, Abrams H, Chan M, Ducharme A, et al. 2017 comprehensive update of the canadian cardiovascular society guidelines for the management of heart failure. Can J Cardiol 2017;33:1342-433

9. Farré N, Vela E, Clèries M, Bustins M, Cainzos-Achirica M, Enjuanes C, et al. Real world heart failure epidemiology and outcome: A populationbased analysis of 88,195 patients. PLoS One 2017;12:e0172745.

10. Chen M, Xue L, Cha C, Liang J, Ma P. Impact of strengthening clinical management and intervention on prognosis and compliance among patients with heart failure. Biomed Res 2017;28:5751-7.

11. Xing W, Lv X, Gao W, Wang J, Yang Z, Wang S, et al. Bone mineral density in patients with chronic heart failure: A meta-analysis. Clin Interv Aging 2018;13:343-53.

12. Schulz E, Arfai K, Liu X, Sayre J, Gilsanz V. Aortic calcification and the risk of osteoporosis and fractures. J Clin Endocrinol Metab 2004;89:4246-53.

13. Marushchak M, Krynytska I, Mikolenko A, Andreychyn Y, Bodnar Y, Chornomydz I. Chronic heart failure causes osteopathy or is osteopathy a factor in development of chronic heart failure? Asian J Pharm Clin Res 2018;11:111-5.

14. Krynytska I, Marushchak M, Zaets T, Savchenko I, Habor H. Investigation of bone mineralization in patients with coronary heart disease complicated by chronic heart failure, stage II-A. Georgian Med News 2017;267:43-8.
15. Smiyan SI, Masik OM,Zhulkevych IV. Indicators of bone mineraldensity of healthy men on the results of dual energy X-ray densitometry. Probl Osteol 2002;2:9-16.

16. Hutomo DI, Masulili SL, Tadjoedin FM, Kusdhany LS. Correlation of serum osteocalcin level and periodontal attachment loss with osteoporosis risk status in postmenopausal women. Int J Appl Pharm 2017;9:92-4.

17. Johnell O, Kanis JA. An estimate of the worldwide prevalence and disability associated with osteoporotic fractures. Osteoporos Int 2006;17:1726-33.

18. Lian XL, Zhang YP, Li X, Jing LD, Cairang ZM, Gou JQ, et al. Exploration on the relationship between the elderly osteoporosis and cardiovascular disease risk factors. Eur Rev Med Pharmacol Sci 2017;21:4386-90.

19. Khrystyna P, Liudmyla P, Halyna S, Miz A, Mariya M, Inna K. Investigation of calcium metabolism in patients with coronary heart disease complicated by chronic heart failure, stage ii-a. Bangladesh J Med Sci 2018;17:395-401

20. Lewiecki EM, Silverman SL. Redefining osteoporosis treatment: Who to treat and how long to treat. Arq Bras Endocrinol Metabol 2006;50:694-704

21. Li S, Ou Y, Zhang H, Zhang Z, Zhou H, Liu L, et al. Vitamin D status and its relationship with body composition, bone mineral density and fracture risk in urban central South Chinese postmenopausal women. Ann Nutr Metab 2014;64:13-9.

22. Farhat GN, Cauley JA. The link between osteoporosis and cardiovascular disease. Clin Cases Miner Bone Metab 2008;5:19-34.

23. Estrada K, Styrkarsdottir U, Evangelou E, Hsu YH, Duncan EL, Ntzani EE, et al. Genome-wide meta-analysis identifies 56 bone mineral density loci and reveals 14 loci associated with risk of fracture. Nat Genet 2012;44:491-501.

24. Bolland MJ, Avenell A, Baron JA, Grey A, MacLennan GS, Gamble GD, et al. Effect of calcium supplements on risk of myocardial infarction and cardiovascular events: Meta-analysis. BMJ 2010;341:c3691.

25. den Uyl D, Nurmohamed MT, van Tuyl LH, Raterman HG, Lems WF. (Sub)clinical cardiovascular disease is associated with increased bone loss and fracture risk; A systematic review of the association between cardiovascular disease and osteoporosis. Arthritis Res Ther 2011;13:R5. 